\title{
Theory of dislocations in diamond and silicon and their interaction with hydrogen
}

\author{
M I Heggie $\dagger$, S Jenkins $\dagger$, C P Ewels $\dagger$, P Jemmer $\dagger$, R Jones $\ddagger$ and P R Briddon§ \\ $\dagger$ CPES, University of Sussex, Falmer, Brighton BN1 9QJ, UK \\ $\$$ School of Physics, University of Exeter, Exeter EX4 4PY, UK \\ $\S$ Department of Physics, University of Newcastle, Newcastle NE1 7RU, UK
}

Received 10 October 2000

\begin{abstract}
Dislocations in semiconductors can be strongly affected by a hydrogen plasma; core states may be passivated and mobility changed. For example, in silicon the activation barrier for dislocation motion drops by $1.0 \mathrm{eV}$ upon exposure to $\mathrm{H}$ plasma for one hour at $470-540{ }^{\circ} \mathrm{C}$. If such an effect were to be found in diamond, a simple scaling argument would yield an activation energy of $1.9 \mathrm{eV}$. Here, density functional calculations have been applied to the $90^{\circ}$ partial dislocation in diamond which confirm this prediction. They also show that, energetically, the soliton model for motion of the $90^{\circ}$ partial is as viable as the strained-bond model.
\end{abstract}

\section{Introduction}

Dislocations in silicon are increasingly important, within industry because of large wafer sizes and strained-layer structures and within the research community as models for plastic behaviour of high-lattice-friction solids. Most of the concepts guiding contemporary work originated 20 to 50 years ago, starting with the initial speculations of Shockley on the shuffle nature of perfect $60^{\circ}$ dislocations and the theoretical work of Hornstra on possible dislocation core structures [1]. TEM observation of dissociated dislocations which moved without constriction [2] indicated that dislocations were most likely in the glide set, which has a low stacking fault energy. In addition to reducing elastic strain energy, the glide set partials lower core energies due to their ability to reconstruct [3-5]. The latest HRTEM experiments confirm the model of glide partials, allowing that one of the partials (the $90^{\circ}$ partial) may have a substantial density of kinks and jogs [6].

In order to explain doping-dependent mobility [12], dislocation motion must at some stage involve electronic states deep in the forbidden gap. Hirsch [3] postulated that these were associated with kinks, Jones [13] that they only existed in saddle points for motion. Viewed by the electron microscope, dislocations show contrast (probably due to long-lived gap states) in the EBIC (electron-beam-induced current) mode for straight segments [14], casting doubt on a 1:1 correspondence between geometrical kinks and dangling bonds. In the case of the strained-bond model, doubts were raised about the possibility of charge-carrier capture within the saddle-point lifetime. While these objections can be overcome by invoking impurity effects for EBIC and/or shallow strain states evolving into the saddle point, they gave impetus to a more robust model in which separate existence for dangling bonds and kinks was postulated. The deep gap states had lifetimes long enough to equilibrate with charge carriers and both straight and curved dislocations could act as recombination centres [15]. The dangling bonds 
arise as phase boundaries between different phases of reconstruction, which may be associated with kinks from time to time, but they do not have identical lifetimes as kinks. They have been variously termed, amongst other things, solitons or anti-phase boundaries and a model for how they facilitate kink nucleation and propagation has been given $[15,16]$. Computational modelling of the energetics and dynamics of these models is now feasible and rapidly expanding (see [7-11] and other articles in this Special Issue).

Dislocation motion in silicon is thermally activated and according to Hirth and Lothe [17] the activation energy, $E$, is the sum of kink formation, $F_{k}$, and migration, $W_{m}$, energies $\left(E=F_{k}+W_{m}\right)$ for segments long enough to guarantee that kink lifetime is limited by kinkkink annihilation. For silicon, there is evidence that $\mathrm{H}$ can reduce $E$ from $2.2 \mathrm{eV}$ to $1.2 \mathrm{eV}$ [18], with a reduction in the prefactor from $7 \times 10^{5} \mathrm{~ms}^{-1}$ to $2 \mathrm{~ms}^{-1}$. How the $\mathrm{H}$ is supplied to the dislocation is uncertain, since the effect depends on a pre-hydrogenation step (typically one hour under $\mathrm{H}$ plasma between 470 and $540{ }^{\circ} \mathrm{C}$ ). We follow Yamashita et al [18] and assume that this step introduces a reservoir of $\mathrm{H}_{\text {or }} \mathrm{H}_{2}$ either in the bulk near the dislocation or in the dislocation core. It should be noted that to date, no direct evidence that hydrogen platelets [19] constitute this reservoir has been found for these samples [37]. Theoretical modelling of the interaction of $\mathrm{H}$ with the $90^{\circ}$ partial in silicon [20] also led to $E=1.2 \mathrm{eV}$.

$\mathrm{H}$ is very important in materials processing. One example of this is proton implantation which is the key step in silicon-on-insulator technology (the so-called 'smart cut' [21]). Another is the $\mathrm{H}$ plasma beam source for the low-pressure growth of diamond and diamondlike carbon (DLC or ta-C:H) [22]. The role of hydrogen is largely to saturate surface carbons and maintain $\mathrm{sp}^{3}$ hybridization, but, depending on the degree of crystalline perfection of the resulting sample and on any applied bias, $\mathrm{H}$ may be trapped in bulk and modify its properties with annealing.

This article is concerned with diamond and its similarity with silicon, commenting briefly on the strained-bond versus the soliton model and presenting results on the effects of $\mathrm{H}$ on mobility of the $90^{\circ}$ dislocation, based on the soliton model.

In silicon, primary dislocations are the $60^{\circ}$ and screw following $\langle 110\rangle$ directions in $\{111\}$ slip planes. These dissociate, respectively, into a $90^{\circ}$ and a $30^{\circ}$ partial and into two $30^{\circ}$ partials also lying in the glide plane [23]. The same appears true for diamond, although the volume of experimental evidence is much smaller [24].

The temperature dependence of creep in IIa and Ia diamonds has been found to vary between $10.7 \mathrm{eV}$ and $14 \mathrm{eV}$ [25], but it is not possible to extract $E$ for a single dislocation. However, $E$ has been calculated using the same methodology as for the $90^{\circ}$ partial in silicon [26] within the strained-bond model [13]. In the case of silicon, $E=1.9 \mathrm{eV}$ was found which is not inconsistent with the measured $E=2.1-2.2 \mathrm{eV}$ for perfect $60^{\circ}$ dislocations in intrinsic silicon. Sitch et al found $E=3.3 \mathrm{eV}$ for diamond [28], i.e. 1.7 times larger than for silicon-a factor which is close to the ratio (1.6) of the cohesive energies of diamond $(7.3 \mathrm{eV})$ and silicon $(4.6 \mathrm{eV})$.

$E=3.3 \mathrm{eV}$ for diamond is consistent with both its hardness and brittleness and the difficulty in producing and moving isolated dislocations in a well controlled experiment. However, another consequence of diamond's strong, short (1.54 $⿱$ ) bonds is that diamond cannot accommodate impurities: there is scant space for substitutional elements from anywhere other than the first row or for interstitials. Segregation energies are high, especially for dislocations, and thus supersaturations of impurities may create and move dislocations. If the hydrogen-enhanced dislocation glide (HEDG) occurs in diamond, scaling $E=1.2 \mathrm{eV}$ for silicon [18] with cohesive energies predicts $E=1.9 \mathrm{eV}$, which might be accessible experimentally. The aim of this work is to investigate the scaled value of $E$ for diamond (hereafter we shall refer to 'scaled energies' which are 1.6 times those for silicon [20]). 


\section{Method}

Here we use a local spin-density approximation (LSDA) code (AIMPRO) [27] applied to 200atom clusters as in the silicon work [20], except that we use a wave-function basis of four s-, $\mathrm{p}_{x^{-}}$, $\mathrm{p}_{y}$ - and $\mathrm{p}_{z}$-symmetry Gaussian functions per carbon atom and four s functions for the valence charge density. This basis set has been successful in describing surface $\mathrm{C}-\mathrm{H}$ reactions in diamond growth, for example, and equivalent calculations of energies in silicon did not change when the basis was increased by extending it with bond-centred orbitals [20]. We apply static structural optimizations via the conjugate gradient method, finding the classical trajectories of the proton and host atoms. Most calculations have been performed on $\mathrm{C}_{134} \mathrm{H}_{98}$ clusters. The exceptions are those performed on the hydrogenated soliton (H-soliton) migration barrier $\left(\mathrm{C}_{104} \mathrm{H}_{82}\right), \mathrm{H}$ in crystalline diamond as $\mathrm{H}_{2}, \mathrm{H}_{2}^{*}$ etc $\left(\mathrm{C}_{95} \mathrm{H}_{86}\right)$ and the soliton pair $\left(\mathrm{C}_{207} \mathrm{H}_{146}\right)$.

As in previous work [28], we study the single-period (SP) structure of the $90^{\circ}$ partial as a model for dislocation motion. It is known for silicon that there are differences between partials in activation energy for motion depending on whether they lead or trail, and on whether it is a $90^{\circ}$ or a $30^{\circ}$ partial [29], but the variations are less than $0.1 \mathrm{eV}$-comparable with LSDA uncertainties and zero-point energies. A similar argument applies to variations between SP and double-period (DP) structures [30,31], which have recently been investigated in diamond [32].

Depending on the reconstruction, a $90^{\circ}$ partial dislocation core has a phase which may be labelled either 'right' (R) or 'left' (L) and the interface between them is the soliton [15]. The soliton necessarily involves a dangling bond, which in chemical terms is a 'free radical' and highly active $[20,33]$. It can migrate along the dislocation line and it can nucleate kink pairs. It binds strongly with $\mathrm{H}$ and this interaction provides an intuitive, and quantitative, model of HEDG in silicon [20]. Figure 1(a) is a glide plane diagram of the soliton after hydrogenation (a soliton-hydrogen complex). Other models, invoking $\mathrm{H}_{2}$ molecules and/or the strained-bond model are possible, but in the first instance we pursue the original soliton model.

\section{Results}

\subsection{Model for a soliton in pure diamond}

We studied the soliton model for a dislocation in pure diamond, calculating the energy of a soliton-anti-soliton pair in a cluster where the soliton and anti-soliton were separated by four reconstruction bonds and did not annihilate spontaneously. We found a soliton formation energy of $2.2 \mathrm{eV}$ (cf. scaled $2.2 \mathrm{eV}$ [20]). The threefold-coordinated atom approaches planar, $\mathrm{sp}^{2}$ hybridization (bond angles $118^{\circ}$ ) - a low-energy configuration for carbon-but we note that the two bonds across the dislocation axis from the dangling bond are extremely stretched (1.7 and $1.8 \AA$ ), presumably due to the greater resistance to bond bending in diamond.

As suggested [15], and found to be the case, for silicon [20], we find that the soliton in diamond is extremely mobile with a migration energy of $0.3 \mathrm{eV}$. (Figure 2, lower curve, shows the barrier located by imposing a single constraint.) Furthermore, it can nucleate a kink pair (as in figure $1(\mathrm{~d})$, omitting the $\mathrm{H}$ atom) with an activation energy of $0.74 \mathrm{eV}$ (cf. scaled $0.45 \mathrm{eV}$ ) and formation energy of $0.23 \mathrm{eV}$ (cf. scaled $0.17 \mathrm{eV}$ ) for the incipient kink pair. The results are summarized in table 1.

Nucleating a kink pair on a straight reconstructed dislocation costs $0.36 \mathrm{eV}$ [28]; thus there is a kink-soliton binding energy of $0.13 \mathrm{eV}$, which is small.

Applying a correction of $0.27 \mathrm{eV}$ for the elastic interaction of kinks within the pair [28] gives a kink formation energy, $F_{k}$, of $0.25 \mathrm{eV}$ in the presence of a soliton. Further, assuming (i) that the barrier to kink formation by solitons is typical of all soliton-catalysed 

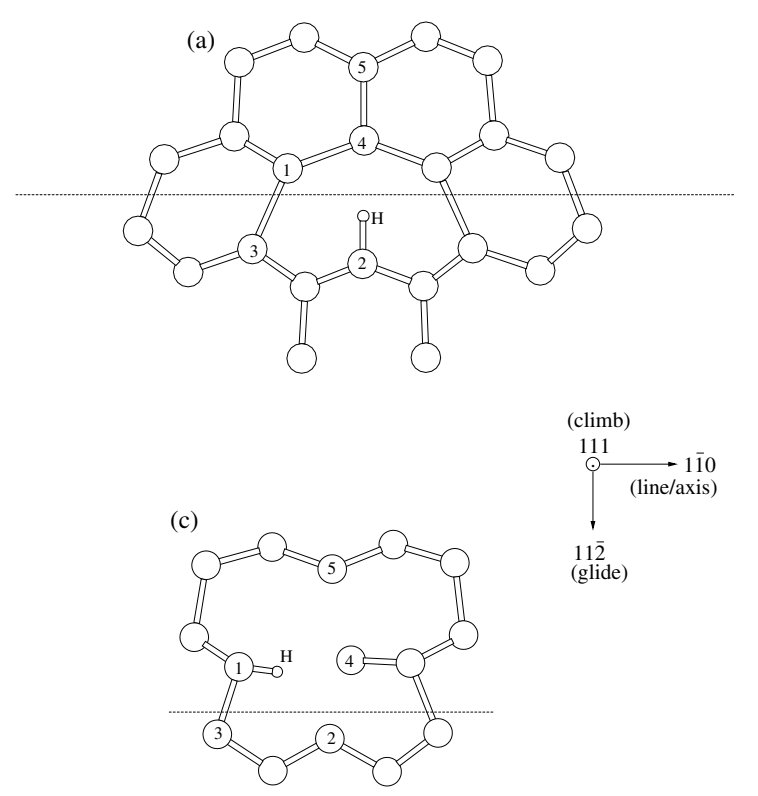

(b)
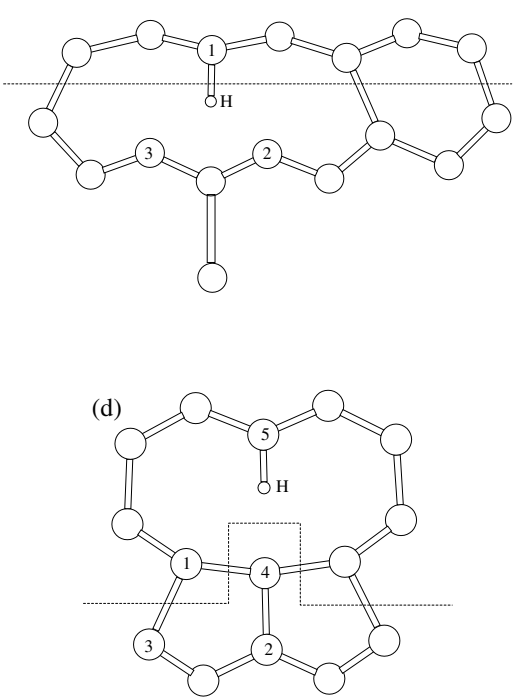

Figure 1. (a) The structure of hydrogenated soliton in a $90^{\circ}$ partial dislocation in diamond; (b) the saddle point for motion along the core; (c) the metastable saddle point for motion perpendicular to the core; (d) the hydrogenated kink pair, $0.5 \mathrm{eV}$ more stable than (a). The numbers are referred to in the text. The plane of the page is (111), left/right is $\langle 1 \overline{1} 0\rangle$, up is $\langle 11 \overline{2}\rangle$. The dislocation axis is shown as a broken line.

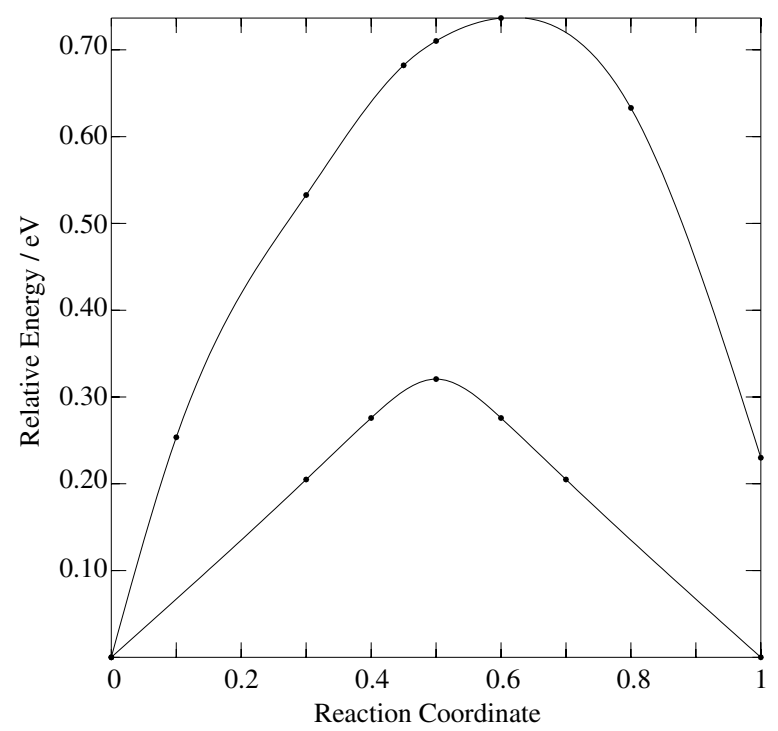

Figure 2. Barriers to soliton motion in a $90^{\circ}$ partial dislocation, along the core (lower curve) and perpendicular to the core forming a kink pair (upper curve).

kink migration steps, (ii) that solitons are in thermal equilibrium and (iii) that the binding between reconstructed kinks and solitons $(0.13 \mathrm{eV})$ is negligible, then a Hirth-Lothe value of $E=2.2+0.25+0.74 \mathrm{eV}=3.19 \mathrm{eV}$ is obtained. As was the case for silicon [20], the 
Table 1. All results are for 231-atom clusters, except that marked with an asterisk, which is for 186-atom clusters. For relative formation energies, a negative value indicates that the structure is more stable. The soliton pair formation energy is $4.4 \mathrm{eV}$, for a $\mathrm{C}_{207} \mathrm{H}_{146}$ cluster with a pair of solitons separated by four reconstruction bonds.

\begin{tabular}{lll}
\hline Structure & \multicolumn{2}{c}{ Energy $(\mathrm{eV})$} \\
\hline Soliton activation barriers & Without $\mathrm{H}$ & With $\mathrm{H}$ \\
Migrating along dislocation core & $0.30(0.24)$ & $1.20(1.65)^{*}$ \\
Initiating kink pair & $0.74(0.45)$ & $1.67(1.82)$ \\
\hline Formation energy compared to soliton & Without H & With H \\
Soliton-kink pair & $0.23(0.17)$ & $-0.50(-0.30)$ \\
\hline
\end{tabular}

soliton model gives $E$ comparable to, but slightly lower than, the strained-bond-model value (3.3 $\mathrm{eV}$ for diamond [28]), illustrating its viability, but the small differences in $E$ between the two models may not be decisive, given the unquantified differences in pre-exponential terms (geometrical and entropy factors). The assumption (ii) that solitons are in thermal equilibrium may not be straightforward, since their production in pairs will cost $4.4 \mathrm{eV}$; however, there are mechanisms (interaction with point defects and impurities) which profoundly reduce this barrier. We assume that these mechanisms are able to maintain the soliton population close to equilibrium, and that the time and space average concentration is given by the Boltzmann factor.

\section{2. $H$ in crystalline diamond}

There are parallels between $\mathrm{H}$ in silicon (see the review [34]) and diamond [35], and in both the neutral ground state is the bond-centred hydrogen atom. However, the reduced bond length in diamond causes two significant inversions of the silicon behaviour. The first is that bondcentred $\mathrm{H}$ is substantially higher in energy $(2.8 \mathrm{eV})$ than an $\mathrm{H}$ atom in the gas phase and the second [38] is that $\mathrm{H}_{2}$ molecules are unstable with respect to the $\mathrm{H}_{2}^{*}$ configuration (a host atom with one $\mathrm{H}$ atom in the bond-centred position and one in the antibonding position). Formation of bond-centred $\mathrm{H}$ requires dilation of the host $\mathrm{C}-\mathrm{C}$ bond by $50 \%$.

\subsection{H interacting with a soliton on a $90^{\circ}$ partial}

Here we discuss a possible $\mathrm{H}$ plasma effect in terms of a flux of $\mathrm{H}$ atoms (i.e. $\mathrm{H}_{\mathrm{BC}}$ ) on the dislocation line, as discussed for the case of silicon. We note that the migration energy of $\mathrm{H}_{\mathrm{BC}}$ is not small and that there are normally large internal stresses and high substrate temperatures involved in diamond or DLC growth. The dislocation has a substantial cross-section from the linear extent of the dilatational part of its strain field and, once trapped at the core, $\mathrm{H}$ atoms, as we will show later, are mobile along the core and therefore must meet; in silicon, they recombine into molecules at high temperatures (the barrier to this process is $0.74 \mathrm{eV}$ ). However, for diamond we have found the barrier to formation of molecules even in the enlarged (sevenfold) dislocation channel to be $5 \mathrm{eV}$.

Our results are summarized in table 1 . First we note that $\mathrm{H}_{\mathrm{BC}}^{0}$ binds strongly to a neutral soliton with an energy of $4.8 \mathrm{eV}$ (giving the structure of figure 1(a)) and to a soliton-kink pair with an energy of $5.3 \mathrm{eV}$ (giving figure 1(d)). Nucleating a kink pair at a free soliton costs $0.23 \mathrm{eV}$, while doing so at a hydrogenated soliton releases $0.50 \mathrm{eV}$, due to reduced steric effects for the $\mathrm{H}$ in the kink pair [36]. Thus, as was the case for silicon, $\mathrm{H}$ flux may be expected to spontaneously nucleate soliton pairs (of energy $4.4 \mathrm{eV}$ ) and, simultaneously, kink pairs, i.e. a 
complex of a hydrogenated soliton with a reconstructed kink pair will be formed in addition to a non-hydrogenated soliton, which will migrate rapidly until it combines with another incoming $\mathrm{H}$ atom.

The steady-state motion of the dislocation will be controlled by the formation and motion of kinks caused by these hydrogenated solitons (figure 1(a)), for which we now calculate barriers to motion and kink formation.

Free-soliton migration was obtained by relaxing all degrees of freedom, subject to a single constraint:

$$
r_{1-3}^{2}-r_{1-2}^{2}=C_{1}
$$

(where $r_{\mathrm{a}-\mathrm{b}}$ is the length of the bond between atoms (a) and (b) from figure 1) and locating the maximum in $E\left(C_{1}\right)$ (which was at $C_{1}=0$ ). With $\mathrm{H}$, additional constraints had to be applied:

$$
\begin{aligned}
& r_{1-3}^{2}-r_{1-2}^{2}=C_{1} \\
& r_{2-\mathrm{H}}^{2}-r_{3-\mathrm{H}}^{3}=C_{2} .
\end{aligned}
$$

Varying $C_{1}$ and $C_{2}$ independently generates a two-dimensional surface (figure 3(a)) from which a barrier (at $\mathrm{D}$ ) of $1.20 \mathrm{eV}$ is deduced. The saddle-point structure for motion along the core (D) consists of $\mathrm{H}$ bonded to the central $\mathrm{C}$ atom number 1 (figure 1(b)).

Kink pair formation involves $\mathrm{H}$-soliton motion perpendicular to the line, via the intermediate structure found for silicon (figure 1(c)). This is an unconstrained meta-stable structure-as it was for silicon. Other points on the left side of this figure were then obtained by interpolating between the intermediate structure and the starting structure. At each step the system was fully relaxed in all directions orthogonal to the translation vector between the initial and intermediate structures. The second half of the motion was similarly determined by applying the constraint of orthogonality to the vector between the intermediate and final structures (figure 1(d)). From figure 3(b) it can be seen that the rate-limiting step for kink pair formation at a hydrogenated soliton will be $1.67 \mathrm{eV}$.

Further expansion of the kink pair can be achieved by similar steps [15]. The ratecontrolling factor will then be the $\mathrm{H}$-catalysed kink migration steps, which experience indicates have activation barriers closely similar to the first step, yielding an overall activation energy for motion of $1.67 \mathrm{eV}$, even lower than the prediction of $1.9 \mathrm{eV}$ obtained by scaling the silicon value with the ratio of diamond:silicon cohesive energies. In the case of silicon, the low prefactor, dependent on the $\mathrm{H}$ concentration, arises from the low mean free path of hydrogenated solitonkink complexes under a substantial flux of $\mathrm{H}$ atoms.

Depending on the flux of $\mathrm{H}$ atoms, the substrate temperature and the presence of traps such as dislocations, it is possible that a model invoking $\mathrm{H}$ dimers, as hydrogenated $\mathrm{C}-\mathrm{C}$ bonds or as $\mathrm{H}_{2}^{*}$ defects, could be appropriate. As for silicon, the molecule will insert exothermically into a reconstruction bond. The pair of $\mathrm{H}$-soliton complexes thus created can move apart and catalyse kink formation and migration (albeit endothermically in the first step).

Two of us (SJ and MIH) have investigated the nature of bonding in these structures and the reasons for the energies obtained [36].

In conclusion, we have shown the soliton mechanism for glide of the $90^{\circ}$ partial dislocation to be viable and we have used it in a model for the effect of $\mathrm{H}$ on the same dislocation, in analogy with an experimentally known effect of $\mathrm{H}$ plasmas on dislocations in silicon. We find, on the basis of activation energies alone, that this effect might lead to experimentally accessible rates of dislocation motion in diamond. There is the possibility that similar reactions and energetics apply to amorphous, dense carbon materials, such as hard a-C:H films. We observe that the temperature range for $\mathrm{HEDG}$ in silicon is $470{ }^{\circ} \mathrm{C}$ to $530{ }^{\circ} \mathrm{C}$; at the same homologous temperatures $\left(T / T_{m}\right)$ in diamond, the corresponding physical temperatures are $1620{ }^{\circ} \mathrm{C}$ to $1770{ }^{\circ} \mathrm{C}$. 
Barrier to $\mathrm{H}-$ soliton motion in a $90^{\circ}$ partial dislocation along the core

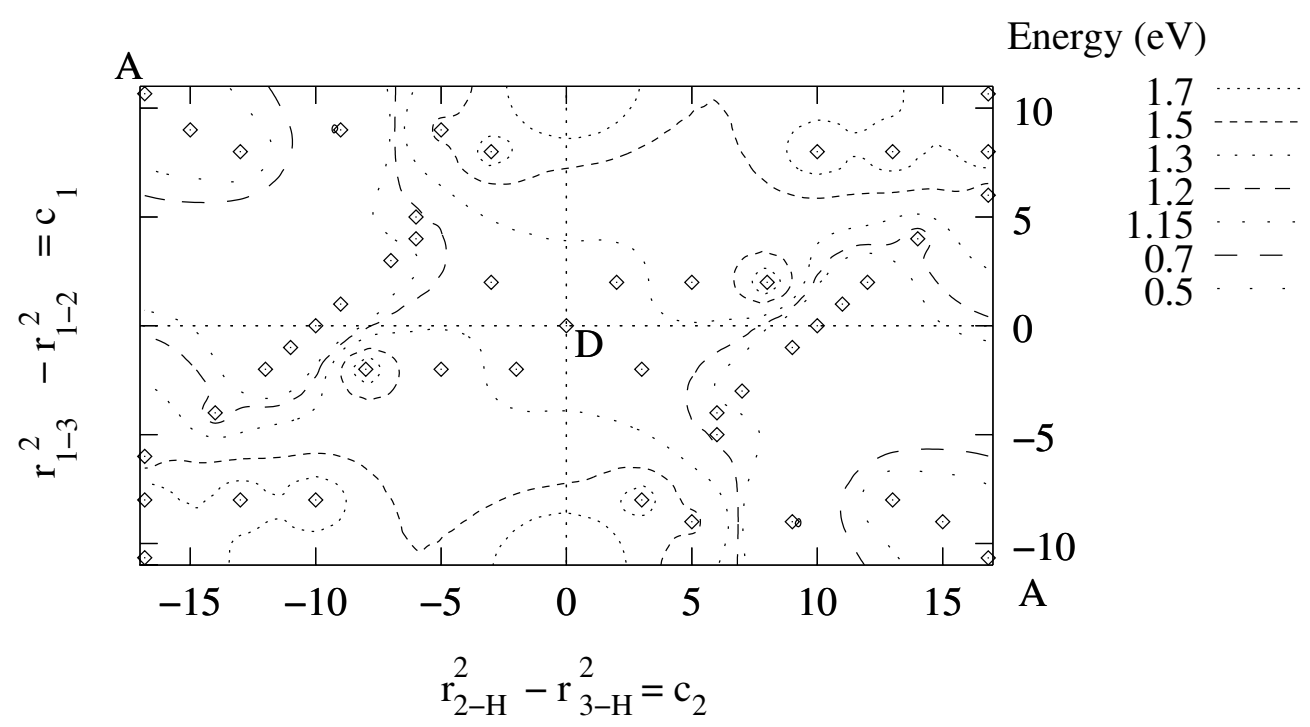

(a)

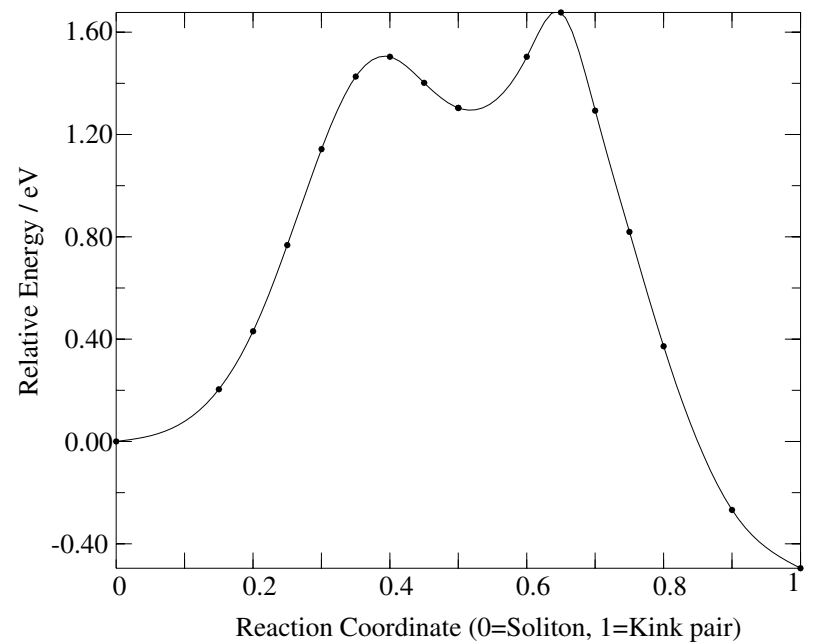

(b)

Figure 3. Barriers to $\mathrm{H}$-soliton motion in a $90^{\circ}$ partial dislocation: (a) along the core (stable $\mathrm{H}$ solitons are top left and bottom right and there is a saddle point at $C_{1}=C_{2}=0$ ); (b) perpendicular to the core forming a kink pair (dots mark points sampled and the curve is to guide the eye).

\section{Acknowledgments}

For computer time we thank the EPSRC (Edinburgh and Manchester Cray T3E) and the Sussex High Performance Computing Initiative. We are indebted to Koji Maeda and Jon Goss for unpublished results. 


\section{References}

[1] Hornstra J 1958 J. Phys. Chem. Solids 5129

[2] Ray I L F and Cockayne D J H 1973 J. Microsc. 98170

[3] Hirsch P B 1979 J. Physique 40 C6 117

Hirsch P B 1980 J. Microsc. 1183

[4] Jones R 1979 J. Physique 40 C6 33

[5] Marklund S 1979 Phys. Status Solidi b 9283

[6] Batson P E 2000 Phys. Rev. B 6116633

[7] Nunes R W, Bennetto J and Vanderbilt D 1998 Phys. Rev. B 5710388

[8] Justo J F, de Koning M, Cai W and Bulatov V V 2000 Phys. Rev. Lett. 842172

[9] Csanyi G, Ismail-Beigi S and Arias T A 1998 Phys. Rev. Lett. 803984

[10] Lehto N and Öberg S 1997 Phys. Rev. Lett. 5612706

[11] Valladares A, White J A and Sutton A P 1998 Phys. Rev. Lett. 814903

[12] George A, Escaravage C, Champier G and Schröter W 1972 Phys. Status Solidi a 53483

[13] Jones R 1980 Phil. Mag. B 42365

[14] Wilshaw P R and Booker G R 1985 Microscopy of Semiconducting Materials 1985 (Inst. Phys. Conf. Ser. 76) (Bristol: Institute of Physics Publishing) p 329

[15] Heggie M I and Jones R 1983 Phil. Mag. B 48365 Heggie M I and Jones R 1983 Phil. Mag. B 48379

[16] Heggie M I 1982 PhD Thesis University of Exeter, UK

[17] Hirth J P and Lothe J 1982 Theory of Dislocations (New York: Wiley) p 531

[18] Yamashita Y, Jyobe F, Kamiura Y and Maeda K 1999 Phys. Status Solidi a 17127

[19] Beaufort M F, Garem H and Lépinoux J 1994 Phil. Mag. A 69881

[20] Ewels C P, Leoni S, Heggie M I, J̈emmer P, Hernandez E, Jones R and Briddon P R 2000 Phys. Rev. Lett. 84690

[21] Bruel M, Aspar B and Auberton Herve A J 1997 Japan. J. Appl. Phys. 361636

[22] Conway N M J, Ferrari A C, Flewitt A J, Robertson J, Milne W I, Tagliaferro A and Beyer W 2000 Diamond Relat. Mater. 9765

[23] Hirsch P B 1985 Mater. Sci. Technol. 1666

[24] Pirouz P, Cockayne D J H, Sumida N, Hirsch P B and Lang A R 1983 Proc. R. Soc. A 386241

[25] Evans T and Sykes J 1974 Phil. Mag. 29135

[26] Öberg S, Sitch P K, Jones R and Heggie M I 1995 Phys. Rev. B 5113138

[27] Jones R and Briddon P R 1998 Identification of Defects in Semiconductors (Semiconductors and Semimetals vol 51A) ed M Stavola (Boston, MA: Academic) ch 6, p 287

[28] Sitch P K, Jones R, Öberg S and Heggie M I 1997 J. Physique III 71381

[29] Wessel K and Alexander H 1977 Phil. Mag. 351523

[30] Bennetto J, Nunes R W and Vanderbilt D 1997 Phys. Rev. Lett. 79245

[31] Lehto N and Öberg S 1998 Phys. Rev. Lett. 805568

[32] Blase X, Lin K R, Canning A, Louie S G and Chrzan D C 2000 Phys. Rev. Lett. 845780

[33] Nunes R W, Bennetto J and Vanderbilt D 1996 Phys. Rev. Lett. 771516

[34] Jones R, Coomer B J, Goss J P, Hourahine B and Resende A 1999 Special Defects in Semiconducting Materials (Solid State Phenomena) (Zurich: Trans. Tech.)

[35] Briddon P R and Jones R 1993 Physica B 185179

[36] Jenkins S and Heggie M I 2000 J. Phys.: Condens. Matter 12

[37] Maeda K 2000 Private communication

[38] Goss J 2000 Private communication 